\title{
Pseudoaneurysm of the Profunda Femoris Artery Following Internal Fixation for Intertrochanteric Fracture: A Report of 2 Cases and Review of the Literature
}

\author{
Miladi M*, Kedous MA, Zaraa M, Lahmar AA, Sayed W and barek MM \\ Department of Orthopedic surgery and Traumatology, Ben Arous Trauma and Burn Center, Tunisia \\ *Corresponding author: Miladi M, Department of Orthopedic surgery and Traumatology, Ben Arous Trauma and Burn Center, Tunisia \\ Submission: 海 February 06, 2019; Published: 侮 February 25, 2019
}

\section{Introduction}

Inter-trochanteric femur fractures are one of the most common fractures among the elderly [1]. With the aging population it has now become one of the most frequent cause of referral in our trauma center in the elderly patients [2]. Their treatment includes a variety of surgical techniques from external fixation to internal fixation with sliding hip screw and mini invasive surgical procedures, like the use of the gamma nail, each having its own indication $[2,3]$. Complications are rare and vascular ones even more [2]. However, in the literature there are an increasing number of publications on this subject [1-8], hence the need to know how to recognize them in order to manage them in time to avoid life-threatening situations, especially in elderly subjects [3]. The published studies are only case descriptions and no real series have been reported to this date.

We also report our experience in two cases with a pseudoaneurysm of the profunda femoris artery as a result of an inter-trochanteric fracture treated with a Dynamic Hip Screw.

\section{Case Description}

In the first case, it was a 67-year-old patient who was brought to our emergency for a right hip trauma following a fall from his height. Clinical examination and radiological exploration concluded to an inter-trochanteric fracture. He was operated on the following day after the trauma and had an internal fixation using a sliding dynamic hip screw. The immediate operative follow-up showed no complications and the patient was discharged after two days under anticoagulant therapy associated with analgesic treatment. Passive rehabilitation of the hip was indicated and partial weight bearing using two axillary crutches was authorized. Monthly clinical and radiological controls did not indicate any particular incidents. However, at three months postoperatively, the patient came to our consultation for a pulsating mass at the level of the right Scarpa triangle. The haemodynamic state of the patient was stable, and the distal pulse was present. Radiography showed a correct consolidation of the fracture. An arterio-venous Doppler ultrasonography was done and showed the presence of a pseudoaneurysm developing from the profunda femoris artery. A further exploration by a CT angiography confirmed the diagnosis. The patient was then taken care of by the vascular surgeons and a resection of the aneurysm was done.

The second case is a 68-year-old diabetic patient who, following a fall from her height, had a complex inter-trochanteric fracture with separation of the two trochanters. The patient had a reduction and osteosynthesis with a dynamic hip screw plate in the days following the trauma. However, the evolution was towards a nonunion of the greater trochanter with an impossibility of walking recovery. The treatment of this non-union with decortication and band wiring was indicated. After surgery the patient began to describe a pulsating swelling, evolving on the inner side of the thigh. Doppler ultrasonography of the thigh was normal; we then pushed the examination to a CT angiography, which revealed that it was a pseudo-aneurysm developed from the profunda femoris artery. The patient was then referred to the vascular surgeons who treated her with a stent.

\section{Discussion}

Inter-trochanteric fractures are one of the most common fractures in the elderly [1]. Their complications are rare and vascular ones exceptional, their incidence varies from $0,08 \%$ to $0,2 \%$ in the different studies, affecting usually the profunda femoris artery but in rare cases even the femoral artery [3,5-8]. Duparc separated these vascular lesions in haemorrhagic ones due to sharp objects from thrombotic ones due to blunt instruments [9]. Pseudoaneurysms are one of these complications [10]. These lesions are usually due to drilling, screw insertion, sometimes to the incorrect introduction of the guide wire in nailing but can also occur by the fracture in itself [3]. Several mechanisms have been incriminated in their genesis: 
a) The irritation of the arterial wall by a pointed instrument (pin, screw, drill, retractor...) represents one of the hypotheses. This is usually a technical error when inserting a too long screw or when a counter-bend or a pin is escaping. This is the most reported mechanism in the literature.

b) Intimate contact between the small, poorly synthesized trochanter and the artery itself may also be irritating.

c) Exaggerated tractions of a curarized patient may result in the rupture of a plaque of atheroma or rupture under adventitious tissue, which will later be responsible of a weakening of the artery wall. The elongation and torsion mechanism is the most incriminated.

d) Deep infections.

e) In addition, an initial post-traumatic rupture of the arterial wall cannot be ruled out.

These lesions could happen at the fracture time, during reduction, internal fixation or even during rehabilitation with the mobilisation of the limb [3]. Many pseudo-aneurysms are ignored because they are spontaneously obliterated [10]. Some authors pinpointed the position of the distal fragment of the femur in adduction and internal rotation as a factor favouring the onset of such lesions [5]. The late discovery of this complication can be explained on the one hand by the deep localization of the artery and on the other hand by the fact that pain, anemia and hematoma may be accounted for by the surgical procedure in itself [1,3]. Clinically it can present itself as a pulsatile mass gradually increasing in size [10]. Moreover, it can simulate a deep venous thrombosis of the lower limb when the hematoma breaks and compresses the veins. Arteriovenous doppler ultrasonography and CT-angiography represent the key examinations in this pathology [3]. The therapeutic options are numerous from ultrasound guided thrombin injection, coil embolisation, vascular surgery and sometimes abstention, each having good results in the literature [3].

\section{Conclusion}

Pseudo aneurysm of the femoral artery represents a rare but well described complication of inter-trochanteric fracture's surgical fixation, its description must be well known by orthopaedic surgeons. In front of a pulsating swelling of the thigh one must think of a pseudo aneurysm and explore it further using Doppler ultrasonography and CT angiography. Its treatment is easy and should be applied as soon as it is discovered to prevent lifethreatening issues.

\section{References}

1. Kim JW, Lee JI, Park KC (2017) Pseudoaneurysm of the deep femoral artery caused by a guide wire following femur intertrochanteric fracture with a hip nail: A case report. Acta Orthopaedica et Traumatologica Turcica 51(3): 266-269.

2. De Raaff C, Van Nieuwenhuizen R, van Dorp T (2016) Pseudoaneurysm after pertrochanteric femur fracture: a case report. Skeletal Radiology 45(4): 575-578.

3. Segal D, Yaacobi E, Marom N, Feldman V, Aliev E, et al. (2017) The incidence of life-threatening iatrogenic vessel injury following closed or open reduction and internal fixation of intertrochanteric femoral factures. International Orthopaedics 41(9): 1845-1850.

4. Thomas M, O'Hara J, Davies A, James SL (2012) Profunda femoris pseudoaneurysm following Birmingham hip resurfacing: an important differential diagnosis for a periarticular cystic mass. Skeletal Radiology 41(7): 853-856.

5. Navarrete F, Longares J (2009) Iatrogenous superficial femoral artery pseudoaneurysm: treatment with a PFNA nail. Spanish Journal of Orthopedic Surgery and Traumatology (English edition), 53(4): 250-253.

6. Toyota T, Horiuchi H, Takizawa T, Yamazaki I, Matsunaga D, et al (2017) A case of femoral pseudoaneurysm after surgery for intertrochanteric fracture. Journal of Orthopaedic Science 22(2): 362-365.

7. Mohan B, Singal S, Bawa AS, Mahindra P, Yamin M (2017) Endovascular management of traumatic pseudoaneurysm: Short \& long term outcomes. Journal of Clinical Orthopaedics and Trauma 8(3): 276-280.

8. Grimaldi M, Courvoisier A, Tonetti J, Vouaillat H, Merloz P (2009) Superficial femoral artery injury resulting from intertrochanteric hip fracture fixation by a locked intramedullary nail. Orthopaedics \& Traumatology: Surgery \& Research 95(5): 380-382.

9. Duparc F (1994) Vascular risk of orthopedic and traumatological surgery. Teaching books of SOFCOT Teaching Conferences Paris, Elsevier, pp. 63-77.

10. Abed YY, Nour K (2017) Neglected false aneurysm of the profunda femoris artery after proximal femoral fracture with failure of fixation and bone erosion: case report and review of the literature. Current Orthopaedic Practice 28(1): 116-123.
Creative Commons Attribution 4.0

International License

For possible submissions Click Here

\section{Submit Article}

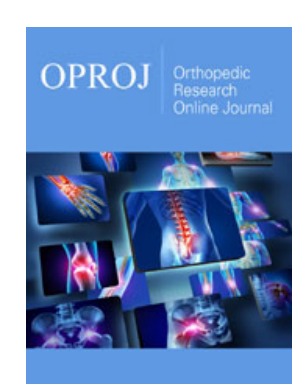

\section{Orthopedic Research Online Journal}

\section{Benefits of Publishing with us}

- High-level peer review and editorial services

- Freely accessible online immediately upon publication

- Authors retain the copyright to their work

- Licensing it under a Creative Commons license

- Visibility through different online platforms 